\title{
miR-330-5p targets SPRY2 to promote hepatocellular carcinoma progression via MAPK/ERK signaling
}

\author{
Shuai Xiao ${ }^{1}$, Mengyuan Yang ${ }^{2}$, Hao Yang ${ }^{1}$, Ruimin Chang ${ }^{1}$, Feng Fang ${ }^{1}$ and Lianyue Yang ${ }^{1}$
}

\begin{abstract}
MicroRNAs (miRNAs) have been identified as critical modulators of cell proliferation and growth, which are the major causes of cancer progression including hepatocellular carcinoma (HCC). Our previous miRNA microarray data have shown that miR-330-5p was always upregulated in HCC. However, the accurate role of miR-330-5p in HCC is still uncertain. Here, we report that miR-330-5p expression is upregulated in HCC tissues and cell lines, and is associated with tumor size, tumor nodule number, capsule formation and Tumor Node Metastasis (TNM) stage in HCC patients. Overexpression of miR-330-5p promotes proliferation and growth of HCC cells in vitro and in vivo, while miR-330-5p knockdown has the inverse effect. Moreover, using miRNA databases and dual luciferase report assay, we find miR-330$5 p$ directly binds to the $3^{\prime}$-untranslated region (3'-UTR) of Sprouty2 (SPRY2). Then we find the novel biofunctional role of SPRY2 inactivation in promoting HCC progression. Finally, we confirm that miR-330-5p suppresses SPRY2 to promote proliferation via mitogen-activated protein kinases (MAPK)/extracellular regulated kinase (ERK) signaling in HCC. Taken together, our findings demonstrate the critical role of miR-330-5p in promoting HCC progression via targeting SPRY2 to activate MAPK/ERK signaling, which may provide a novel and promising prognostic marker and therapeutic target for HCC.
\end{abstract}

\section{Introduction}

Hepatocellular carcinoma (HCC) is one of the most common and lethal malignancies in the world, which accounts for about $50 \%$ of the total number of cases and deaths each year in China ${ }^{1}$. Although surgical and systemic treatments for $\mathrm{HCC}$ have improved greatly in the past decade, the long-term survival is still poor due to the rapid cancer progression ${ }^{2}$. Cancer cell uncontrolled proliferation is one of the fundamental factors for $\mathrm{HCC}$ progression $^{3}$. Evidence have shown that various growth factors, PTEN, p53, and etc. are responsible for HCC proliferation, and a series of targeted drugs such as sorafenib, APR-246, AD80 and so on are developed

Correspondence: Lianyue Yang (lianyueyang@hotmail.com)

'Liver Cancer Laboratory, Xiangya Hospital, Central South University, Changsha, Hunan, China

${ }^{2}$ Department of Obstetrics and Gynecology, The Second Xiangya Hospital of Central South University, Changsha, Hunan, China

These authors contributed equally: Shuai Xiao, Mengyuan Yang, Hao Yang accordingly ${ }^{4}$. However, the intervention effect of these drugs is still unsatisfactory due to the precise regulatory mechanisms of HCC proliferation remain poorly understood. Therefore, there is an emergent need to reveal the potential mechanism of HCC proliferation and develop the new effective molecular targeted therapeutic strategy for HCC progression.

MicroRNAs (miRNAs) are a class of small noncoding RNAs that negatively modulate gene expression, which attract widespread attention and become a hotspot in cancer research ${ }^{5}$. More importantly, miRNA expression profiles have been successfully used to classify different tumor stages or subsets with distinct molecular pathology characteristic $^{6}$. Our previous study has found a specific HCC subtype named solitary large HCC (SLHCC), which was one single lesion with a diameter over $5.0 \mathrm{~cm}$ and grew expansively, with aintact capsule or pseudo-capsule. The long-term survival of SLHCC was significantly longer than nodular HCC (NHCC, node number more than 1)

\section{(c) The Author(s) 2018}

(c) (i) Open Access This article is licensed under a Creative Commons Attribution 4.0 International License, which permits use, sharing, adaptation, distribution and reproduction c. in any medium or format, as long as you give appropriate credit to the original author(s) and the source, provide a link to the Creative Commons license, and indicate if changes were made. The images or other third party material in this article are included in the article's Creative Commons license, unless indicated otherwise in a credit line to the material. If material is not included in the article's Creative Commons license and your intended use is not permitted by statutory regulation or exceeds the permitted use, you will need to obtain permission directly from the copyright holder. To view a copy of this license, visit http://creativecommons.org/licenses/by/4.0/. 


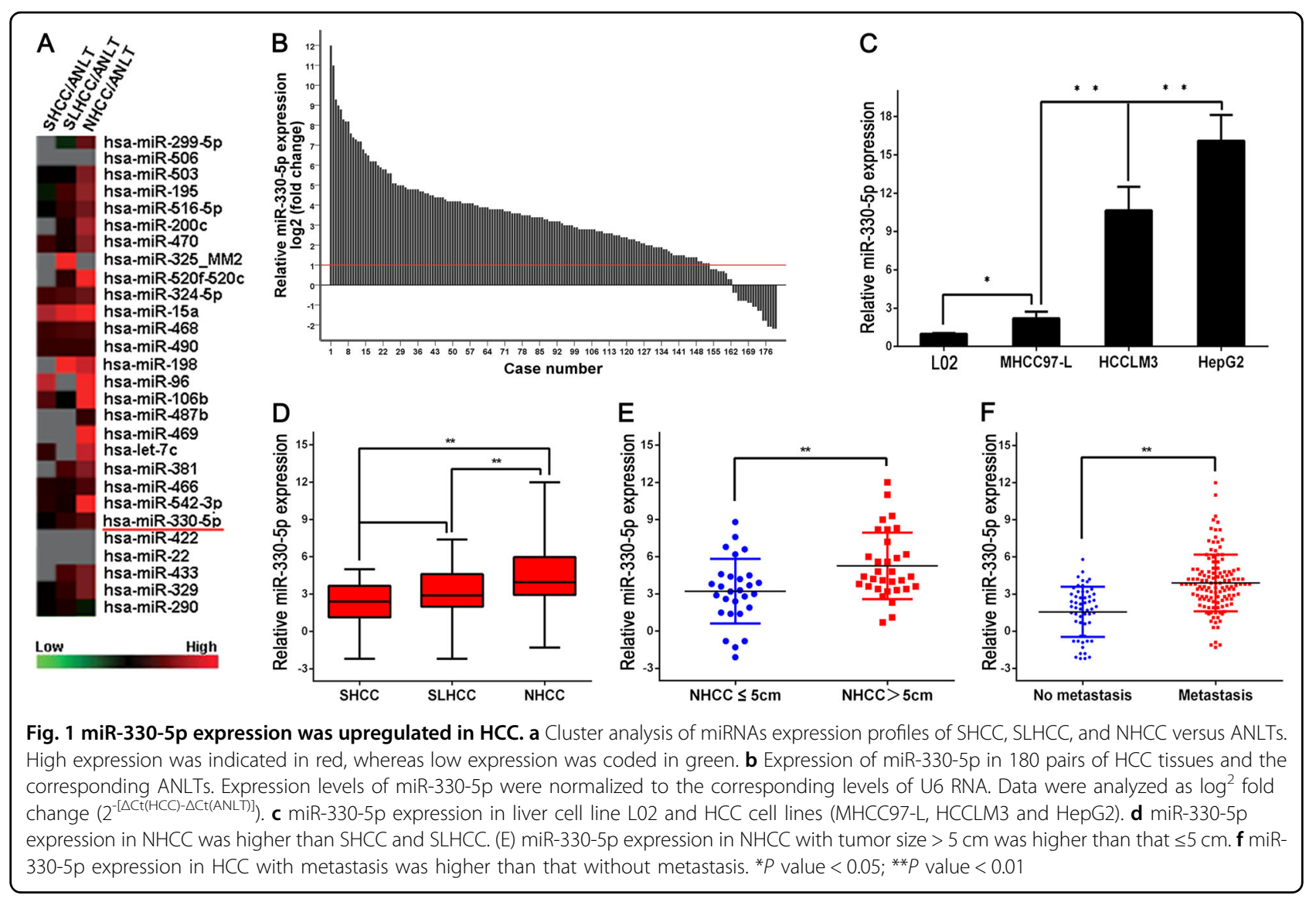

and similar with small HCC (SHCC, 1 node, diameter $\leq$ $5.0 \mathrm{~cm})^{7,8}$. Accordingly, our previous genomic analysis revealed that the three $\mathrm{HCC}$ subtypes had distinct gene expression profiles, such as EGFL7, WAVE2, and VMP1 which could significantly affect tumor growth and metastasis $^{7-11}$. These findings suggested that these HCC subtypes might have different inherent molecular characteristics, as well as miRNAs characteristic patterns. To identify this, we have detected the miRNA expression patterns in HCC by miRNA array analysis in the previous study $^{12}$. Results showed some miRNAs had remarkably different expression levels among the HCC subtypes, such as miR-140-5p, miR-188-5p, miR-331-3p, etc., and these miRNAs had a strong effect on HCC progression and prognosis $^{12-14}$.

Besides these reported miRNAs, we also found miR330-5p expression level was upregulated in HCC, and the expression level in NHCC was significantly higher than SHCC and SLHCC in the miRNA array. miR-330-5p is the mature sequence of miR-330, and increasing studies reported that miR-330 played an important role in tumor progression such as glioblastoma, prostate cancer, as well as $\mathrm{HCC}^{15-17}$. However, there is still no report about the role of miR-330-5p in HCC now. Given the importance of miR-330 in cancer progression and miR-330-5p expression pattern in $\mathrm{HCC}$, thereby there is a strong demand to explore the role of miR-330-5p in HCC progression.

In this study, we provide the first evidence that miR330-5p is upregulated in HCC and its high expression level related to poor survival of HCC patients. miR-330-5p overexpression promotes HCC proliferation and growth through targeting Sprouty2 (SPRY2) mediated mitogenactivated protein kinase (MAPK)/extracellular regulated kinase (ERK) signaling. These results suggest that miR330-5p may be a valuable prognostic biomarker and potential therapeutic target for HCC.

\section{Results}

miR-330-5p expression was significantly upregulated in HCC

Our previous miRNA expression microarray analysis showed that miR-330-5p was higher expressed in HCC compared to ANLT, especially step-increased in SHCC, SLHCC, and NHCC (Fig. 1a). We then performed realtime polymerase chain reaction (PCR) to detect miR-3305 p expression in 180 pairs of HCC (60 pairs of each HCC subtype) bulk tissues and corresponding ANLTs. Consistent with the microarray results, miR-330-5p expression was significantly up-regulated in HCC tissues 
compared to corresponding ANLTs (more than 2-fold [i.e., $\log _{2}$ (fold change) $>1$ ]) (Fig. 1b). Then, we also detected miR-330-5p expression in liver cell lines L02 and HCC cell lines (MHCC97-L, HCCLM3, and HepG2) by real-time PCR. Results showed that miR-330-5p expression was markedly higher in $\mathrm{HCC}$ cell lines than liver cell line L02, and the expression level was the highest in HepG2 cells which had strong proliferation capacity, but the lowest in MHCC97-L cells whose proliferation capacity was relatively weak (Fig. 1c). Subsequently, we made a comparison of miR-330-5p expression levels in different HCC subtypes, the results showed that miR-330-5p expression in NHCC was significantly higher than that in SHCC and SLHCC $(P<0.01$, respectively) (Fig. 1d). Because miR-330-5p expression in SHCC was similar to SLHCC (Fig. 1d), which indicated size was not a critical factor for solitary tumor. Next, we compared miR-330-5p expression in NHCC with different sizes, results showed that miR-330-5p expression in group of NHCC with tumor size $>5.0 \mathrm{~cm}$ was obviously higher than that $\leq$ $5.0 \mathrm{~cm}(P<0.01$, Fig. 1e). Moreover, we also found miR330-5p expression in HCC with metastasis was higher than that without metastasis $(P<0.01$, Fig. 1f). These results showed that miR-330-5p expression was upregulated in HCC tissues and cell lines, especially in NHCC, NHCC with bigger tumor size and HCC with metastasis, which implied that miR-330-5p expression might be associated with $\mathrm{HCC}$ progression and prognosis.

\section{miR-330-5p high expression indicated poor prognosis of $\mathrm{HCC}$}

To further explore the correlation of miR-330-5p expression with clinicopathologic features and long-term survival of HCC patients after hepatic resection (HR), the median relative expression level of miR-330-5p in all 180 HCC samples was chosen as the cut-off point for separating HCC with the low and high expression of miR-330$5 p$ groups. Results showed that high miR-330-5p expression was significantly associated with tumor size, tumor nodule number, capsule formation, Tumor Node Metastasis (TNM) stage and Barcelona Clinic Liver Cancer (BCLC) stage in HCC (all $P<0.05$, Table 1). Kaplan-Meier survival analysis revealed that the 1, 3, 5year overall survival (OS) and disease-free survival (DFS) of low miR-330-5p expression group were 95.6, 71.7, $53.3 \%$, and 91.1, 68.1, 48.9\%, which were significantly better than that in high miR-330-5p expression group of 67.8, 26.1, 14.1\% and 55.6, 23.6, 11.8\% $(P<0.001$, respectively, Fig. 2a). Further stratification analysis revealed that OS and DFS among the three HCC subtypes were different. NHCC had the poorer OS when compared with SHCC and SLHCC $(P=0.001$ and $P=0.027)$ as well as in DFS $(P<0.001$ and $P=0.014)$, but the OS $(P=$ $0.242)$ and DFS $(P=0.209)$ were similar between SHCC
Table 1 Correlations between miR-330-5p expression level and clinicopathological variables of $\mathbf{1 8 0}$ cases of HCC

\begin{tabular}{lll}
\hline Clinicopathologic variables $n \quad \begin{array}{l}\text { miR-330-5p } \\
\text { expression }\end{array}$ & $P$ value \\
& Low (90) High (90) \\
\hline
\end{tabular}

\begin{tabular}{|c|c|c|c|c|}
\hline \multicolumn{5}{|l|}{ Gender } \\
\hline Male & 131 & 69 & 62 & \\
\hline Female & 49 & 21 & 28 & 0.241 \\
\hline \multicolumn{5}{|l|}{ Age(years) } \\
\hline$\leq 60$ & 146 & 77 & 69 & \\
\hline$>60$ & 34 & 13 & 21 & 0.128 \\
\hline \multicolumn{5}{|l|}{$A F P, n g / m L$} \\
\hline$<20$ & 87 & 48 & 39 & \\
\hline$\geq 20$ & 93 & 42 & 51 & 0.180 \\
\hline \multicolumn{5}{|c|}{ Hepatitis B status } \\
\hline Negative & 16 & 10 & 6 & \\
\hline Positive & 164 & 80 & 84 & 0.295 \\
\hline \multicolumn{5}{|l|}{ Cirrhosis } \\
\hline Absence & 51 & 29 & 22 & \\
\hline Presence & 129 & 61 & 68 & 0.247 \\
\hline \multicolumn{5}{|l|}{ Liver function } \\
\hline Child-Pugh A & 161 & 79 & 82 & \\
\hline Child-Pugh B & 19 & 11 & 8 & 0.467 \\
\hline \multicolumn{5}{|l|}{ Tumor size $(\mathrm{cm})$} \\
\hline$\leq 5$ & 68 & 41 & 27 & \\
\hline$>5$ & 112 & 49 & 63 & 0.031 \\
\hline \multicolumn{5}{|c|}{ Tumor nodule number } \\
\hline Solitary & 113 & 64 & 49 & \\
\hline Multiple( $\geq 2)$ & 67 & 26 & 41 & 0.021 \\
\hline \multicolumn{5}{|c|}{ Capsule formation } \\
\hline Presence & 82 & 51 & 31 & \\
\hline Absence & 98 & 39 & 59 & 0.003 \\
\hline \multicolumn{5}{|c|}{ Edmondson-Steiner grade } \\
\hline$|-| \mid$ & 93 & 51 & 42 & \\
\hline III-IV & 87 & 39 & 48 & 0.180 \\
\hline \multicolumn{5}{|c|}{ Microvascular invasion } \\
\hline Absence & 84 & 38 & 46 & \\
\hline Presence & 96 & 52 & 44 & 0.232 \\
\hline \multicolumn{5}{|l|}{ TNM stage } \\
\hline Stage I & 75 & 47 & 28 & \\
\hline Stage II-III & 105 & 43 & 62 & 0.004 \\
\hline \multicolumn{5}{|l|}{ BCLC stage } \\
\hline Stage 0 -A & 87 & 51 & 36 & \\
\hline Stage B-C & 93 & 39 & 54 & 0.025 \\
\hline
\end{tabular}

No patients with Child-Pugh C or TNM Stage IV were included AFP alpha-fetoprotein, HBsAg, TNM tumor node metastasis, BCLC Barcelona Clinic Liver Cancer

and SLHCC (Fig. 2b). These results were consistent with our previous study about the outcome of the different HCC subtypes ${ }^{7}$. In this study, we also found NHCC group had more percent of high miR-330-5p expression cases than SHCC and SLHCC groups (all $P<0.01$, Fig. 2c), while SHCC and SLHCC groups had no significant difference $(P>0.05$, Fig. $2 \mathrm{c})$, which was consistent with Fig. 1d. Stratification survival analysis also showed that HCCs 


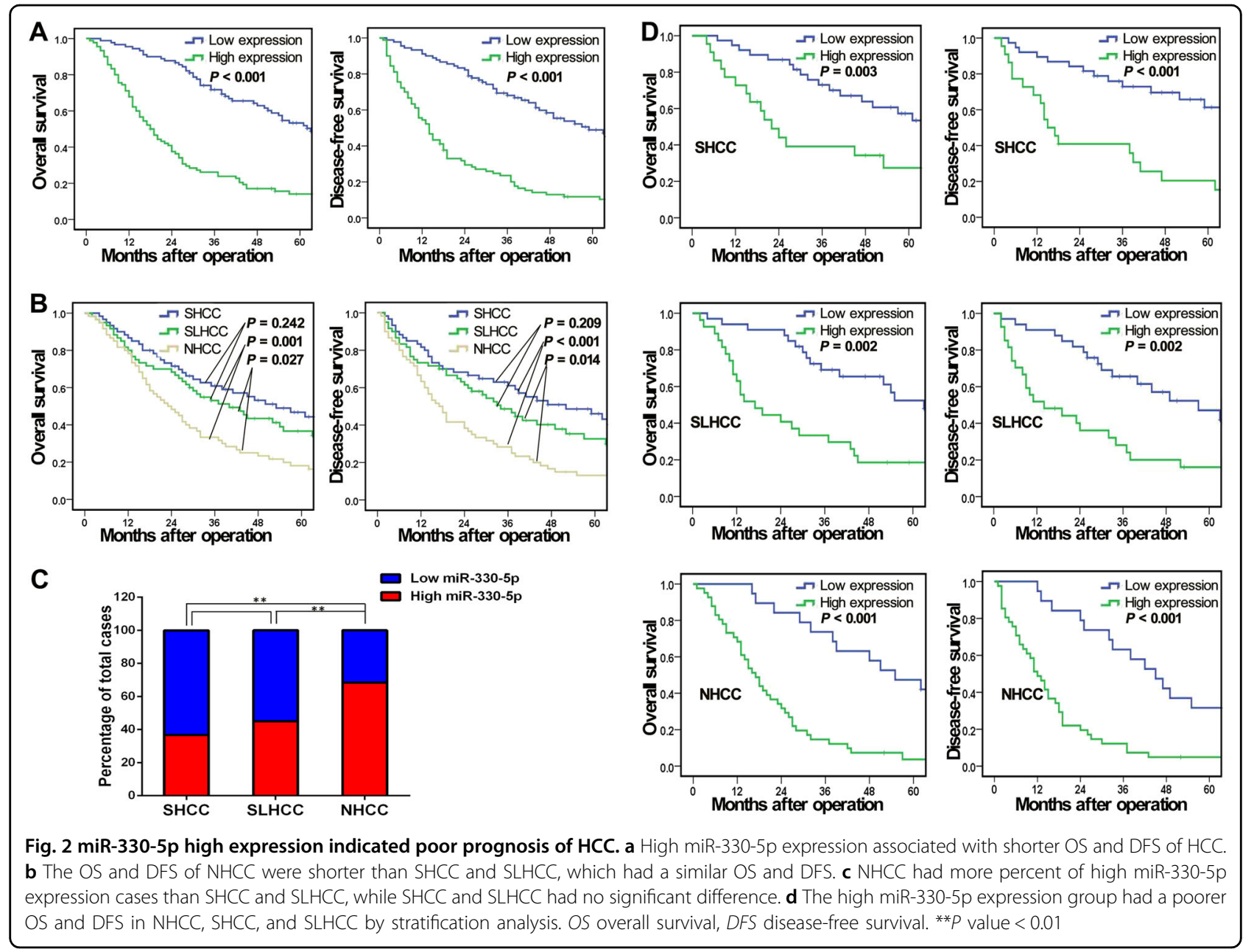

with high miR-330-5p expression group had a poorer OS and DFS than that with low miR-330-5p expression in each of the three HCC subtypes (all $P<0.01$, Fig. $2 \mathrm{~d}$ ). These data indicated that miR-330-5p expression was associated with poor prognosis of HCC and might be a stratification prognostic marker for HCC subtypes.

Next, in order to assess the value of the miR-330-5p expression in predicting HCC prognosis, the Cox proportional hazards regression model was applied. The univariate analysis revealed that cirrhosis, tumor size, tumor nodule number, Edmondson-Steiner grade, microvascular invasion, TNM stage, BCLC stage, as well as miR-330-5p expression were significant for OS (all $P<$ 0.05 , Table 2). Next, multivariate analysis revealed that OS was significantly dependent on cirrhosis, microvascular invasion, BCLC stage, and miR-330-5p expression (all $P<$ 0.05 , Table 2). Further analysis of DFS demonstrated that cirrhosis, tumor nodule number, microvascular invasion, TNM stage, BCLC stage, and miR-330-5p expression were significant in the univariate analysis (all $P<0.05$, Table 3), while only tumor nodule number, microvascular invasion, BCLC stage, and miR-330-5p expression were independent risk factors for DFS in multivariate analysis (all $P<0.05$, Table 3). These results indicated that miR330-5p expression was closely correlated with adverse clinicopathological features and shorter survival of HCC patients, and was a potential prognostic marker for HCC.

\section{miR-330-5p promoted proliferation and growth of HCC}

Because the rapid growth of tumor is the main cause of poor prognosis, we then performed miR-330-5p gain-andloss-of-function assays to explore the role of miR-330-5p in HCC. We chose HepG2 to establish stable infection of anti-miR-330-5p cells (designed as HepG2 $2^{\text {anti-miR-330-5p }}$ ) and selected MHCC97-L to construct stable ectopic expression miR-330-5p cells (designed as MHCC97-L ${ }^{\text {miR- }}$ 330-5p) for next functional assays. The interfered efficiency was determined by real-time PCR (Fig. 3a). Results showed the proliferation capacity of HepG2 and MHCC $97-L$ cells was obviously changed by miR-330-5p interference, but invasion potential had no significant change (Supplementary Fig. 1a, b). The methyl thiazolyl 
Table 2 The Cox regression analyses of overall survival (OS) and miR-330-5p expression level as well as clinicopathological parameters

\begin{tabular}{|c|c|c|c|c|c|}
\hline \multirow{2}{*}{ Variables } & \multirow[t]{2}{*}{$n$} & \multicolumn{2}{|c|}{ Univariable analysis } & \multicolumn{2}{|c|}{ Multivariable analysis } \\
\hline & & HR $(95 \% \mathrm{Cl})$ & $P$ & $\mathrm{HR}(95 \% \mathrm{Cl})$ & $P$ \\
\hline \multicolumn{6}{|l|}{ Gender } \\
\hline Male & 131 & 1 & & & \\
\hline Female & 49 & $1.31(0.43-2.27)$ & 0.427 & NA & \\
\hline \multicolumn{6}{|l|}{ Age(years) } \\
\hline$\leq 60$ & 146 & 1 & & & \\
\hline$>60$ & 34 & $1.21(0.71-1.83)$ & 0.548 & NA & \\
\hline \multicolumn{6}{|l|}{ AFP, $n g / m L$} \\
\hline$<20$ & 87 & 1 & & & \\
\hline$\geq 20$ & 93 & $1.84(0.54-3.23)$ & 0.085 & NS & \\
\hline \multicolumn{6}{|c|}{ Hepatitis B status } \\
\hline Negative & 16 & 1 & & & \\
\hline Positive & 164 & $1.53(0.49-2.86)$ & 0.143 & NA & \\
\hline \multicolumn{6}{|l|}{ Cirrhosis } \\
\hline Absence & 51 & 1 & & 1 & \\
\hline Presence & 129 & $3.42(1.59-5.73)$ & $<0.001$ & $2.94(1.36-4.82)$ & 0.001 \\
\hline \multicolumn{6}{|l|}{ Liver function } \\
\hline Child-Pugh A & 161 & 1 & & & \\
\hline Child-Pugh B & 19 & $1.93(0.78-3.46)$ & 0.072 & NS & \\
\hline \multicolumn{6}{|l|}{ Tumor size $(\mathrm{cm})$} \\
\hline$\leq 5$ & 68 & 1 & & & \\
\hline$>5$ & 112 & $2.01(1.06-3.73)$ & 0.036 & NS & \\
\hline \multicolumn{6}{|c|}{ Tumor nodule number } \\
\hline Solitary & 113 & 1 & & & \\
\hline Multiple( $\geq 2)$ & 67 & $2.23(1.17-3.92)$ & 0.014 & NS & \\
\hline \multicolumn{6}{|c|}{ Capsule formation } \\
\hline Presence & 82 & 1 & & & \\
\hline Absence & 98 & $1.62(0.56-3.04)$ & 0.183 & NA & \\
\hline \multicolumn{6}{|c|}{ Edmondson-Steiner grade } \\
\hline$|-| \mid$ & 93 & 1 & & & \\
\hline III-IV & 87 & $1.79(1.15-2.90)$ & 0.042 & NS & \\
\hline \multicolumn{6}{|c|}{ Microvascular invasion } \\
\hline Absence & 84 & 1 & & 1 & \\
\hline Presence & 96 & $3.72(1.63-6.51)$ & $<0.001$ & $2.85(1.29-4.68)$ & 0.002 \\
\hline \multicolumn{6}{|l|}{ TNM stage } \\
\hline Stage $|-| \mid$ & 75 & 1 & & & \\
\hline Stage III & 105 & $2.62(1.44-4.23)$ & 0.009 & NS & \\
\hline \multicolumn{6}{|l|}{ BCLC stage } \\
\hline Stage $0-A$ & 87 & 1 & & 1 & \\
\hline Stage B-C & 93 & $2.91(1.25-5.03)$ & $<0.001$ & $2.46(1.18-4.05)$ & 0.013 \\
\hline \multicolumn{6}{|c|}{ MiR-330-5p expression } \\
\hline Low & 90 & 1 & & 1 & \\
\hline High & 90 & $2.14(1.18-3.25)$ & 0.007 & $1.96(1.07-3.24)$ & 0.028 \\
\hline
\end{tabular}

$H R$ hazard risk ratio, $\mathrm{Cl}$ confidence interval, NA not applicable, NS not significant

tetrazolium (MTT) assay and colony formation assay showed that HepG $2^{\text {anti-miR-330-5p }}$ cells had lower proliferation rate and fewer colony numbers than control cells, but MHCC97-L miR-330-5p cells had increased proliferation rate and more colony numbers than control cells (Fig. 3b, c). The 5-Ethynyl-2'-deoxyuridine (EdU) assays also showed the percentage of positive cells was lower in HepG2 $2^{\text {anti-miR-330-5p }}$ compared with control cells, while in MHCC97-L ${ }^{\text {miR-330-5p }}$ cells had the opposite effect (Fig. 3d). Then, the cell-cycle analysis further validated that anti-miR-330-5p increased the percentage of HepG2 cells in G1 phase, but decreased the percentage of cells in $S$ phase, while ectopic expression miR-330-5p decreased the percentage of MHCC97-L cells in G1 phase and increased the percentage of cells in $S$ phase (Fig. 3e). These data concluded that miR-330-5p could increase the proliferation capacity of $\mathrm{HCC}$ cell in vitro.

We further verified the functional role of miR-330-5p in the mouse model. HepG2 $2^{\text {anti-miR-330-5p }}$ MHCC97-L ${ }^{\text {miR- }}$ $330-5 p$, and their control cells were injected into the bilateral inguinal region of nude mice respectively, and tumor growth was evaluated. Results showed that subcutaneous tumors generated from HepG2 $2^{\text {anti-miR-330-5p }}$ cells grew slower and smaller than control cells, while tumors from MHCC97-L miR-330-5p cells grew quicker and bigger than control cells (Fig. 3f). Further, we established liver orthotopic HCC mouse model and detected by in vivo imaging system (IVIS) to test the oncogenic function of miR-330-5p in vivo. Consistent with the in vitro and subcutaneous model data, results showed that HepG2 $2^{\text {anti-miR-330-5p }}$ cells generated smaller tumors than control cells, while MHCC97-L $\mathrm{L}^{\text {miR-330-5p }}$ cells generated bigger tumors than control cells (Fig. 3g). Taken these results together, we could draw the conclusion that miR$330-5 p$ promoted HCC growth in vitro and in vivo.

\section{SPRY2 was a direct target of miR-330-5p in HCC}

To explore the potential mechanism of miR-330-5p promotes the growth of HCC, we first searched the validated miR-330-5p associated pathways by miRWalk 2.0 (Supplementary Table 1), and focused in MAPK signaling for the strong effect in promoting proliferation. Then we searched candidate targets for miR-330-5p in miRWalk (TargetScan, miRanda, DIANAmT and PICTAR5 databases) (Supplementary Table 2). Among the hundreds of candidates, we focused the genes which were closely related to MAPK signaling and chose SPRY2 as the potential target of miR-330-5p which was predicted by all the 4 databases. SPRY2 was an important inhibitor for ERK, which was the central member of the canonical MAPK pathway and played a crucial role in promoting cell proliferation, especially in cancers ${ }^{18}$. Therefore, we focus on SPRY2 as the potential target of miR-330-5p for further study.

To validate whether miR-330-5p could directly bind to the $3^{\prime}$ UTR of SPRY2, we constructed the luciferase reporter vector of the SPRY2 WT 3' UTR and the mutant 3' UTR target sequences (Fig. 4a). The real-time PCR and western blot assays also confirmed that SPRY2 expression was upregulated in HepG2 $2^{\text {anti-miR-330-5p }}$ cells but decreased in MHCC97-L ${ }^{\text {miR-330-5p }}$ cells (Fig. 4b, c). As expected, the relative SPRY2-3'UTR WT luciferase 


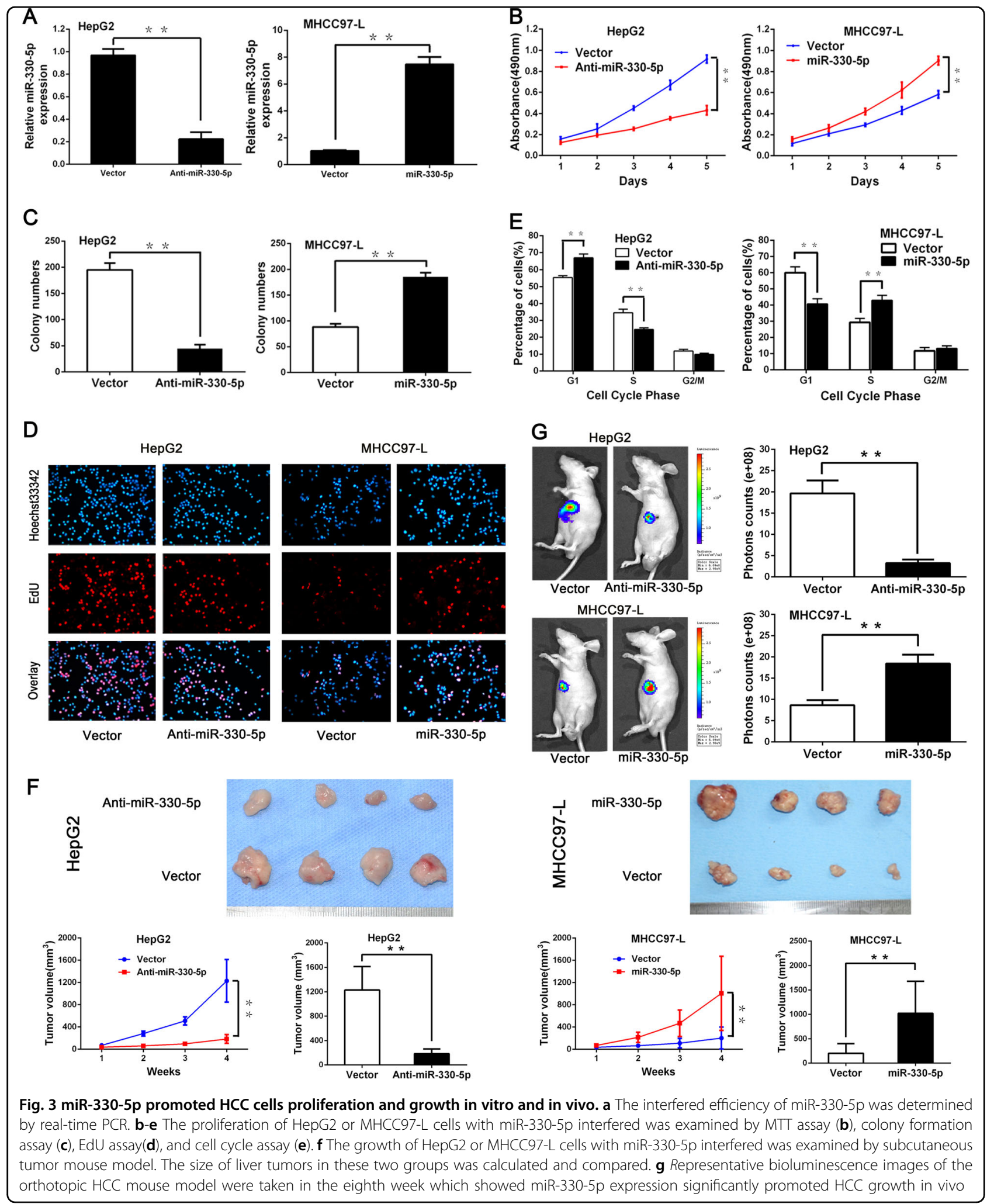

activity was significantly decreased in $293 \mathrm{~T}$ cells and MHCC97-L cells after transfected with miR-330-5p, but increased in HepG2 ${ }^{\text {Anti-miR-330-5p }}$ cells which had miR- 330-5p knockdown (Fig. 4d). Then we detected the SPRY2 expression in HCC samples, results showed the SPRY2 expression was down-regulation in most of $\mathrm{HCCs}$, which 
Table 3 The Cox regression analyses of disease-free survival (DFS) and miR-330-5p expression level as well as clinicopathological parameters

\begin{tabular}{|c|c|c|c|c|c|}
\hline \multirow[t]{2}{*}{ Variables } & \multirow[t]{2}{*}{$n$} & \multicolumn{2}{|c|}{ Univariable analysis } & \multicolumn{2}{|c|}{ Multivariable analysis } \\
\hline & & HR $(95 \% \mathrm{Cl})$ & $P$ & HR $(95 \% \mathrm{Cl})$ & $P$ \\
\hline \multicolumn{6}{|l|}{ Gender } \\
\hline Male & 131 & 1 & & & \\
\hline Female & 49 & $1.17(0.63-1.85)$ & 0.736 & NA & \\
\hline \multicolumn{6}{|l|}{ Age(years) } \\
\hline$\leq 60$ & 146 & 1 & & & \\
\hline$>60$ & 34 & $1.41(0.74-2.31)$ & 0.529 & NA & \\
\hline \multicolumn{6}{|l|}{ AFP, ng/mL } \\
\hline$<20$ & 87 & 1 & & & \\
\hline$\geq 20$ & 93 & $1.75(0.59-3.24)$ & 0.092 & NS & \\
\hline \multicolumn{6}{|c|}{ Hepatitis B status } \\
\hline Negative & 16 & 1 & & & \\
\hline Positive & 164 & $2.03(0.87-3.45)$ & 0.068 & NS & \\
\hline \multicolumn{6}{|l|}{ Liver function } \\
\hline Child-Pugh A & 51 & 1 & & & \\
\hline Child-Pugh B & 129 & $1.88(0.69-3.27)$ & 0.081 & NS & \\
\hline \multicolumn{6}{|l|}{ Cirrhosis } \\
\hline Absence & 161 & 1 & & & \\
\hline Presence & 19 & $1.95(1.12-3.16)$ & 0.034 & NS & \\
\hline \multicolumn{6}{|c|}{ Tumor size (cm) } \\
\hline$\leq 5$ & 68 & 1 & & & \\
\hline$>5$ & 112 & $1.52(0.48-2.79)$ & 0.233 & NA & \\
\hline \multicolumn{6}{|c|}{ Tumor nodule number } \\
\hline Solitary & 113 & 1 & & 1 & \\
\hline Multiple( $\geq 2)$ & 67 & $2.13(1.26-3.43)$ & 0.010 & $1.97(1.15-3.04)$ & 0.028 \\
\hline \multicolumn{6}{|c|}{ Capsule formation } \\
\hline Presence & 82 & 1 & & & \\
\hline Absence & 98 & $1.79(0.72-3.17)$ & 0.069 & NS & \\
\hline \multicolumn{6}{|c|}{ Edmondson-Steiner grade } \\
\hline $\mathrm{H}-\mathrm{II}$ & 93 & 1 & & & \\
\hline III-IV & 87 & $1.44(0.83-2.24)$ & 0.325 & NA & \\
\hline \multicolumn{6}{|c|}{ Microvascular invasion } \\
\hline Presence & 84 & 1 & & 1 & \\
\hline Absence & 96 & $2.47(1.22-4.57)$ & 0.006 & $2.12(1.15-3.82)$ & 0.013 \\
\hline \multicolumn{6}{|l|}{ TNM stage } \\
\hline Stage I-II & 75 & 1 & & & \\
\hline Stage III & 105 & $1.89(1.07-3.21)$ & 0.035 & NS & \\
\hline \multicolumn{6}{|l|}{$\mathrm{BCLC}$ stage } \\
\hline Stage 0-A & 87 & 1 & & 1 & \\
\hline Stage B-C & 93 & $2.61(1.34-4.74)$ & 0.001 & $2.25(1.16-3.92)$ & 0.007 \\
\hline \multicolumn{6}{|c|}{ MiR-330-5p expression } \\
\hline Low & 90 & 1 & & 1 & \\
\hline High & 90 & $2.17(1.21-3.31)$ & 0.004 & $1.93(1.13-3.24)$ & 0.019 \\
\hline
\end{tabular}

was significantly inversely correlated with the expression of miR-330-5p by Spearman rank correlation (Fig. 4e, $r=$ $-0.429, P<0.001)$. We further detected the SPRY2 expression in the tumor of HepG2 $2^{\text {anti-miR-330-5p }}$ cells by immunohistochemical staining, the representative images showed the markedly increased positive staining of SPRY2 compared with control tumor (Fig. 4f). We also analyzed the correlation of SPRY2 expression with HCC patients' survival, results showed that low SPRY2 expression was associated with poorer OS and DFS $(P<0.01$, respectively), which was consistent with high miR-330-5p in HCC (Fig. 4g). These results suggested that SPRY2 was the direct downstream target and negatively modulated by miR-330-5p in HCC.

\section{miR-330-5p suppressed SPRY2 expression to promote HCC growth}

Further assays to validate the functional role of miR330-5p suppressing SPRY2 in HCC were carried out. We constructed SPRY2 overexpression and knockdown plasmid vector respectively, then transfected the SPRY2 overexpression plasmid into MHCC97-L miR-330-5p cells (MHCC97-L ${ }^{\text {miR-330-5p+SPRY2 }}$, and SPRY2 knockdown plasmid into HepG2 $2^{\text {anti-miR-330-5p }}$ (HepG2 $2^{\text {anti-miR-330-5p }}$ + shSPRY2 $)$ cells. The intervention efficiency was assessed by real-time PCR and western blot (Supplementary Fig. 2a, b). The MTT and colony formation assays revealed that SPRY2 overexpression inhibited the proliferation of HepG2 and MHCC97-L ${ }^{\text {miR-330-5p }}$ cells, while silence SPRY2 significantly recovered the proliferation ability of HepG2 $2^{\text {anti-miR-330-5p }}$ and MHCC97-L cells (Figs. 5a, b). The EdU and cell cycle assays result further verified these results (Figs. $5 \mathrm{c}, \mathrm{d}$ ). Then, subcutaneous in vivo assays showed that tumors generated from HepG2 $2^{\text {anti-miR-330-5p }}$ + shSPRY2 2 cells were bigger than tumors from HepG2 ${ }^{\text {anti- }}$ miR-330-5p cells, while tumors generated from MHCC97$\mathrm{L}^{\text {miR-330-5p+SPRY2 }}$ cells were smaller than tumors from MHCC97-L ${ }^{\text {miR-330-5p }}$ cells (Fig. 5e). Next, the tumors from orthotopic HCC model also showed the similar results by IVIS (Fig. $5 \mathrm{f}$ ).

These gain-and-loss-of-function data from in vitro and in vivo assays showed that miR-330-5p suppressed SPRY2 expression to promote HCC growth.

\section{miR-330-5p suppressed SPRY2 to promote HCC growth via MAPK/ERK signaling}

After we confirmed that miR-330-5p could repress SPRY2 expression to promote the growth of HCC, we next wanted to explore the potential regulatory network. Because SPRY2 was generally considered inhibiting MAPK/ERK signaling, we first detected ERK and p-ERK expression in miR-330-5p interfered cells by western blot. Results showed that miR-330-5p overexpression was associated with high p-ERK expression, while inhibition miR-330-5p expression was associated with low p-ERK expression, but the total ERK expression had no significant change (Fig. 6a). Then we detected the ERK and p-ERK expression in SPRY2-interfered cells and found that SPRY2 expression was negatively correlated with pERK expression (Fig. 6b). These data indicated that miR- 


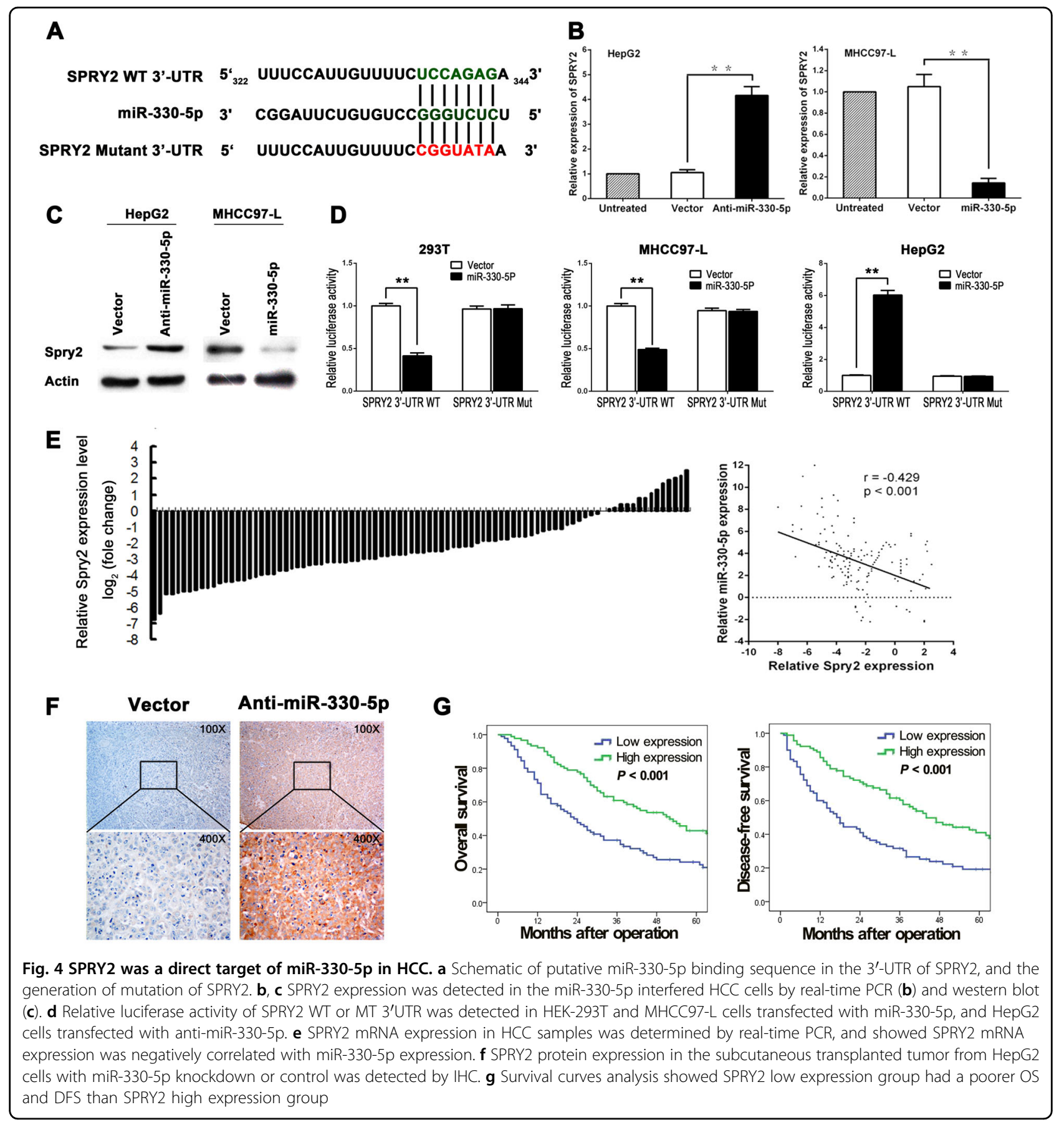

330-5p might regulate ERK signaling activity via SPRY2. We further studied the role of SPRY2 on the central members of ERK signaling. Results showed that SPRY2 overexpression inhibited the expressions of c-RAF, pMEK and p-ERK in MHCC97-L ${ }^{\text {miR-330-5p }}$ cells, while SPRY2 silence recovered the expression of c-RAF, p-MEK and $p$-ERK in HepG2 $2^{\text {anti-miR-330-5p }}$ cells, and had no obvious effect on K-RAS and MEK (Fig. 6c). These data further indicated that SPRY2 modulated the activity of
ERK signaling via c-RAF and then induced the phosphorylation of MEK and ERK. Besides these, we detected the expression of SPRY2, c-RAF, p-MEK and p-ERK in xenografts from SPRY2-interfered HCC cells by IHC. Results also showed in SPRY2 low expression HCC tissues always had high c-RAF, p-MEK and p-ERK expression, while in SPRY2 low expression HCC tissues had the low c-RAF, p-MEK, and p-ERK expression (Fig. 6d). Taken together, we concluded that miR-330-5p suppressed 


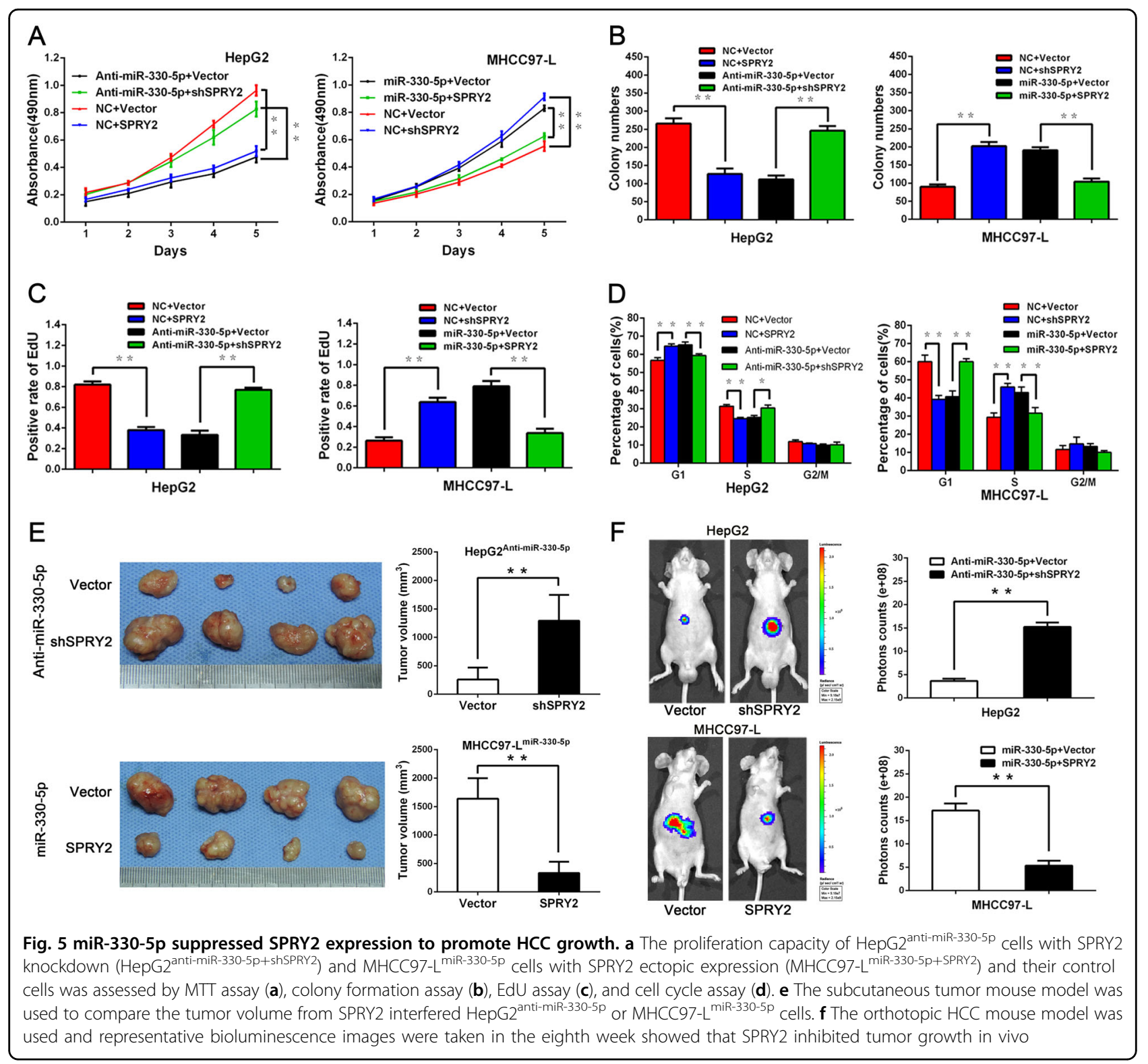

SPRY2 to promote proliferation via activating c-RAFMEK-ERK signaling in HCC (Fig. 6e).

\section{Discussion}

Uncontrollable cell proliferation is one of the fundamental hallmarks of cancer progression ${ }^{3}$. Increasing studies have demonstrated that miRNAs played a crucial role in cancer cell proliferation ${ }^{5}$. Consistent with these, our previous miRNAs profiling has found that miR-140-5p, miR-331-3p, miR-188-5p and etc. had a great effect on HCC proliferation, growth, progression and correlated with poor prognosis of HCC patients ${ }^{12-14}$. In this study, we first found and confirmed that miR-330-5p significantly promoted HCC proliferation, growth, and progression in vitro and in vivo, which was not reported before. And these findings further verified the important value of miRNAs in HCC progression.

miR-330-5p and miR-330-3p were the mature sequences generated from $5^{\prime}$ or $3^{\prime}$ terminal of miR-330 premiRNA. miR-330 aberrant expression was closely related to cancer development and progression, such as glioblastoma, colorectal cancer, prostate cancer, as well as $\mathrm{HCC}^{15-17,19}$. However, the exact role of miR-330 in cancer was still controversial, and so was miR-330-5p. Some studies found miR-330-5p functioned as a tumor suppressor in pancreatic cancer, colorectal cancer and glioblastoma, while our study indicated the oncogenic role in HCC which was consistent with the previous study of miR-330 in $\mathrm{HCC}^{15,20-22}$. Therefore, our findings suggested that both miR-330 and miR-330-5p were 

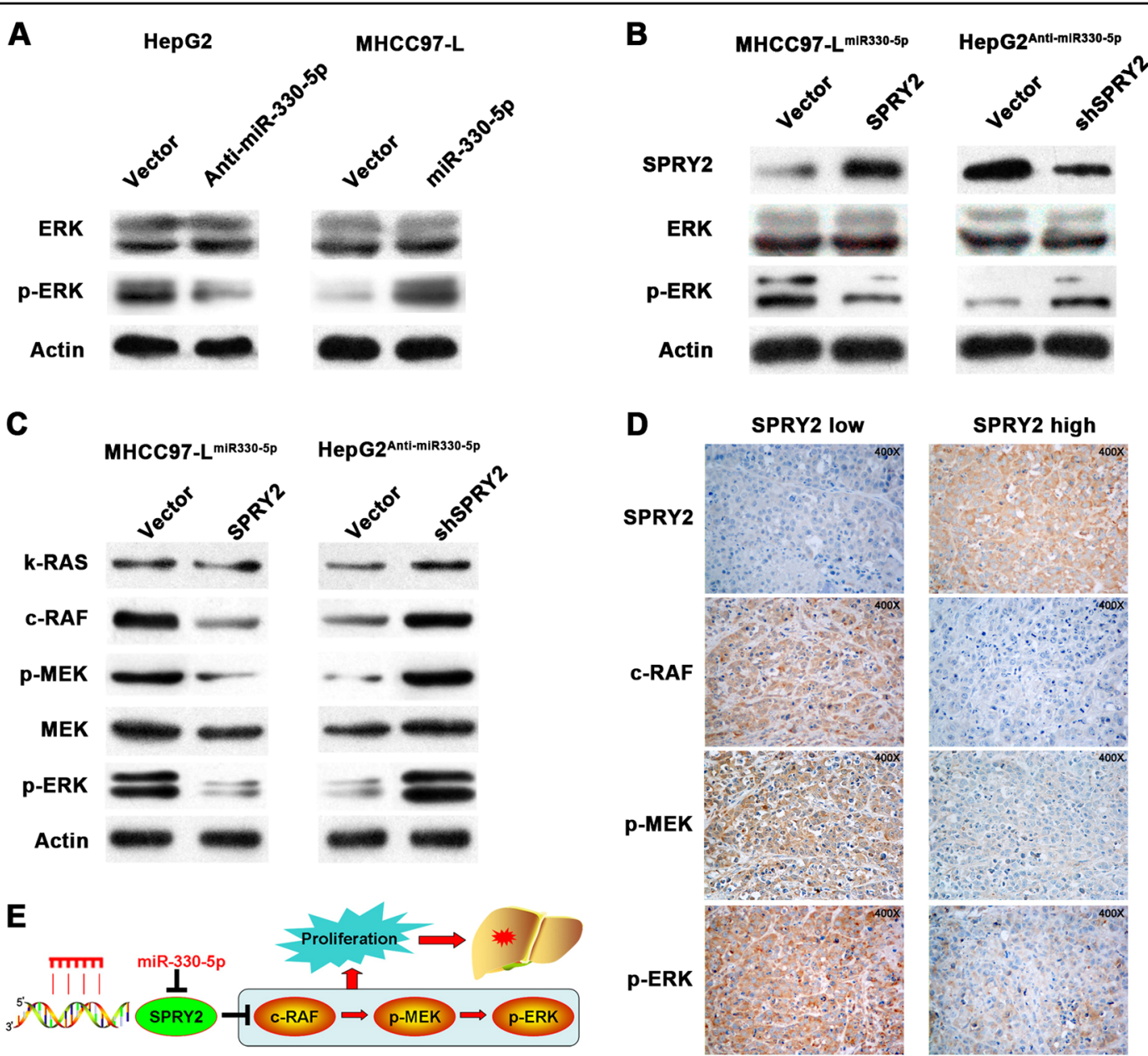

Fig. 6 miR-330-5p suppressed SPRY2 to promote HCC growth via MAPK/ERK signaling. a ERK and p-ERK expression in miR-330-5p interfered cells were detected by western blot. $\mathbf{b}$ ERK and $p$-ERK expression level were detected in SPRY2 interfered HepG2 ${ }^{\text {anti-miR-330-5p }}$ or MHCC97-L ${ }^{\text {miR-330-5p }}$ cells by western blot. $\mathbf{c}$ The central members of MAPKJERK signaling ( $k$-RAS, c-RAF, p-MEK, p-ERK and MEK) were detected by western blot. $\mathbf{d} c-R A F, \mathrm{p}-$ MEK and p-ERK protein expressions in subcutaneous transplanted tumor with high or low SPRY2 expression were detected by IHC. e The diagram of miR-330-5p targeted SPRY2 and activated MAPK/ERK signaling to promote HCC growth and progression

functioned as oncogenic miRNAs in HCC, which expanded our understanding of miR-330 in cancer.

More interestingly, we found miR-330-5p expression levels were similar in SHCC and SLHCC which had obviously different tumor sizes, but miR-330-5p expression level was elevated in NHCC with the increase of size. These data seemed to be a contradiction at first view, but we thought that explained why SHCC and SLHCC were different to NHCC. The potential explanation was that SHCC and SLHCC had relatively low metastasis potential and the tumor size of them might just reflect the growth time but not invasion ability ${ }^{23-25}$. However, NHCC had an obvious higher aggressive capacity and tumor size was a marker of aggressive potential, thus miR-330-5p expression level in NHCC was elevated with increased tumor size. These findings were also consistent with our previous studies that size of the solitary tumor was not a determining factor for the staging of HCC, which was also supported by some other studies ${ }^{7,26}$.

In this study, we have explored the molecular mechanism of miR-330-5p in promoting HCC progression. We found and verified SPRY2 was the direct target, SPRY2 was generally considered as an inhibitor of MAPK/ ERK signaling and played an important role in embryonic development and stemness maintenance ${ }^{27-29}$. Recent years, researchers had further recognized that SPRY2 had a great capacity in regulating cell proliferation, survival, migration, and angiogenesis, thus became a hotspot in cancer progression ${ }^{30,31}$. Existing studies showed that SPRY2 inactivation promoted various cancer development and progression, such as melanoma, lymphoma, gastric cancer ${ }^{32-34}$. Since Fong first reported that SPRY2 was down-regulated and inhibited proliferation in $\mathrm{HCC}$ in 2006, there were many studies confirmed that SPRY2 
inactivation was powerful for hepatocarcinogenesis, but no study involved in HCC progression ${ }^{35,36}$. Based on this, our study had not only validated the previous studies but also further indicated that SPRY2 inactivation by miR330-5p promoted HCC progression and predicted poor prognosis. Therefore, our findings provided novel clues of SPRY2 in HCC progression and might develop a novel biomarker for HCC surveillance and treatment.

The potential molecular mechanism of SPRY2 inactivation was also studied in this study. Numerous studies had confirmed that SPRY2 played its biologic role mainly through inhibiting the canonical MAPK/ERK signaling, which was composed and consecutively activated by EGFR, RAS, RAF, MEK, and ERK ${ }^{37}$. In this study, we found SPRY2 regulated the expression of c-RAF, p-MEK, and p-ERK in HCC, but had no effect on k-RAS, MEK and ERK. Therefore, SPRY2 might exert its ERK inhibitor role via suppressing c-RAF in HCC, which were consistent with prior studies in leukemia ${ }^{38,39}$. RAF-MAPK/ERK was an important signaling for targeting drugs such as regorafenib and sorafenib, thus our findings potentially provided a new clue for developing anovel therapeutic approach for $\mathrm{HCC}^{40}$.

In summary, our study has well defined the oncogenic role and promising prognostic factor of miR-330-5p in HCC. We have verified miR-330-5p directly targeted SPRY2 to activate MAPK/ERK signaling to promote HCC progression, which provided an important evidence that targeting miR-330-5p would be the promising therapeutic approach for HCC treatment.

\section{Materials and methods}

\section{HCC patients, samples, and data}

One hundred and eighty randomly group selected patients (60 SHCC, 60 SLHCC, and 60 NHCC) who underwent $\mathrm{HR}$ for $\mathrm{HCC}$ and had detailed clinicopathological and prognostic data at Department of Surgery, Xiangya Hospital of Central South University (Changsha, China) from January 2007 to December 2010 were included in this study. Only patients with initially diagnosed with HCC were enrolled, and none of them accepted chemotherapy, transcatheter arterial chemoembolization or radiotherapy before the operation. Diagnosis of HCC was confirmed by two independent histopathologists blind to the information of patients. The surgical indication was HCC patient with enough residual liver volume, and lack of distant metastasis, decompensated cirrhosis, and organic dysfunction. The snapfrozen and paraffin embedded HCCs and ANLTs were collected and reserved. Tumor recurrence and metastasis were detected by serum AFP, ultrasonography and chest $\mathrm{x}$-ray every 3 months in the first 2 years, and twice a year in the next 5 years. Contrast-enhanced Computed Tomography scan or Magnetic Resonance Imaging was performed if relapse or metastasis was suspected clinically. The OS defined as the time from HR to death for $\mathrm{HCC}$ or to the date of the last follow-up; DFS defined as the time from the date of HR to the date when relapse or metastasis was detected. The complete clinical, pathological and prognosis data of these patients were collected and stored in our database by a researcher fellow. The present prognostic study strictly follows the consensus of the Reporting Recommendations for Tumor Marker Prognostic Studies (REMARK) guideline ${ }^{41}$. All the human materials and matching information (all anonymous) were obtained with a written informed consent form, and approved by the ethics committee of Xiangya Hospital of Central South University. All the experiments and procedures were in accordance with the Helsinki Declaration.

\section{In vivo assay}

First, luciferase-labeled HepG2 and MHCC97-L cell lines are built via transfecting by recombinant plasmid pSin-hyg-GFP/luc2 (Berthold, Bad Wildbad, Germany). Then, the HCC subcutaneous and orthotopic transplantation model in nude mice were constructed as described before $^{12}$. Briefly, $5 \times 10^{6}$ treated cells were subcutaneously injected into the left buttock regions of 4 nude mouse (randomly selected, 3-4 weeks of age, male, BALB/c), and the untreated cells were injected into the right buttock of the same mouse as a control. After 4 weeks of implantation, the mice were sacrificed, and the subcutaneous tumor size was calculated and recorded used vernier caliper as follows: tumor volume $\left(\mathrm{mm}^{3}\right)=\left(\mathrm{L} \times \mathrm{W}^{2}\right) / 2$, where $\mathrm{L}=$ long axis and $\mathrm{W}=$ short axis $^{42}$. All the measurements were repeated three times. Then the subcutaneous tumor was cut into pieces of the size as $1 \mathrm{~mm}^{3}$ and implanted into the liver of each group (also random selected, 4 in each group). The activity of luciferase was detected by IVIS Lumina II (Xenogen, Hopkinton, MA) every week according to the protocol of IVIS. After 8 weeks of implantation, the mice were sacrificed, and the size of tumors was calculated and compared as mentioned above. Serial sections were subjected to IHC staining. The animal management and experimental procedures were carried out in accordance with the Guidelines of National Institutes of Health for the Care and Use of Laboratory Animals, and approved by the Medical Experimental Animal Care Commission of Central South University.

\section{Statistical analysis}

Statistical analyses were performed using SPSS 18.0 for Windows (SPSS Inc., Chicago, IL) and GraphPad Prism6 (GraphPad Software Inc., La Jolla, CA). Data were expressed as the mean \pm standard error of the mean from at least three independent experiments. Quantitative data between groups were compared using the Student $t$ test or analysis of variance. Dichotomous variables were analyzed 
by the $\chi^{2}$ test or Fisher exact test. Correlations between different protein expressions level were analyzed using Spearman's rank analysis. Overall survival and diseasefree survival curves were obtained by the Kaplan-Meier method, and differences were compared by the log-rank test. Univariate analysis and multivariate analysis were analyzed with Cox proportional hazard regression model to verify the independent risk factors. A $P$-value $<0.05$ was considered statistically significant.

\section{Acknowledgements}

This work was supported by the National Key R\&D Program of China (2016YC0902403), the National Science \& Technology Major Projects (2012ZX100020122011), Specialized Research Fund for Doctoral Program of Higher Education of China (20130162130007), National Nature Science Foundation of China (81502539), and Key Project of National Nature Science Foundation of China (81330057).

\section{Conflict of interest}

The authors declare that they have no conflict of interest.

\section{Publisher's note}

Springer Nature remains neutral with regard to jurisdictional claims in published maps and institutional affiliations.

Supplementary Information accompanies this paper at (https://doi.org/ 10.1038/s41389-018-0097-8).

Received: 25 July 2018 Revised: 24 September 2018 Accepted: 18 October 2018

Published online: 21 November 2018

\section{References}

1. Torre, L. A. et al. Global cancer statistics, 2012. CA Cancer J. Clin. 65, 87-108 (2015).

2. El-Serag, H. B. Hepatocellular carcinoma. N. Engl. J. Med. 365, 1118-1127 (2011).

3. Hanahan, D. \& Weinberg, R. A. Hallmarks of cancer: the next generation. CELL 144, 646-674 (2011).

4. Forner, A., Llovet, J. M. \& Bruix, J. Hepatocellular carcinoma. Lancet $\mathbf{3 7 9}$ 1245-1255 (2012).

5. Ventura, A. \& Jacks, T. MicroRNAs and cancer: short RNAs go a long way. Cell 136, 586-591 (2009).

6. Giordano, S. \& Columbano, A. MicroRNAs: new tools for diagnosis, prognosis, and therapy in hepatocellular carcinoma? Hepatology 57, 840-847 (2013).

7. Yang, L. Y. et al. Solitary large hepatocellular carcinoma: a specific subtype of hepatocellular carcinoma with good outcome after hepatic resection. Ann. Surg. 249, 118-123 (2009)

8. Wang, W. et al. Genomic analysis reveals RhoC as a potential marker in hepatocellular carcinoma with poor prognosis. Br. J. Cancer 90, 2349-2355 (2004).

9. Wang, W., Wu, F., Fang, F., Tao, Y. \& Yang, L. Inhibition of invasion and metastasis of hepatocellular carcinoma cells via targeting RhoC in vitro and in vivo. Clin. Cancer Res. 14, 6804-6812 (2008).

10. Wang, W., Wu, F., Fang, F., Tao, Y. \& Yang, L. RhoC is essential for angiogenesis induced by hepatocellular carcinoma cells via regulation of endothelial cell organization. Cancer Sci. 99, 2012-2018 (2008).

11. Wu, F. et al. Novel role for epidermal growth factor-like domain 7 in metastasis of human hepatocellular carcinoma. Hepatology 50, 1839-1850 (2009).

12. Yang, H., Fang, F., Chang, R. \& Yang, L. MicroRNA-140-5p suppresses tumor growth and metastasis by targeting transforming growth factor beta receptor 1 and fibroblast growth factor 9 in hepatocellular carcinoma. Hepatology $\mathbf{5 8}$ 205-217 (2013).
13. Fang, F. et al. MicroRNA-188-5p suppresses tumor cell proliferation and metastasis by directly targeting FGF5 in hepatocellular carcinoma. J. Hepatol. 63, 874-885 (2015).

14. Chang, R. M., Yang, H., Fang, F., Xu, J. F. \& Yang, L. Y. MicroRNA-331-3p promotes proliferation and metastasis of hepatocellular carcinoma by targeting $\mathrm{PH}$ domain and leucine-rich repeat protein phosphatase. Hepatology 60, 1251-1263 (2014).

15. Hu, X., Feng, Y., Sun, L., Qu, L. \& Sun, C. Roles of microRNA-330 and its target gene ING4 in the development of aggressive phenotype in hepatocellular carcinoma cells. Dig. DIS SCl 62, 715-722 (2017).

16. Li, Y., Zhu, X., Xu, W., Wang, D. \& Yan, J. miR-330 regulates the proliferation of colorectal cancer cells by targeting Cdc42. BIOCHEM BIOPH RES CO 431 560-565 (2013)

17. Lee, K. H. et al. MicroRNA-330 acts as tumor suppressor and induces apoptosis of prostate cancer cells through E2F1-mediated suppression of Akt phosphorylation. Oncogene 28, 3360-3370 (2009).

18. Samatar, A. A. \& Poulikakos, P. I. Targeting RAS-ERK signalling in cancer: promises and challenges. Nat. Rev. Drug. Discov. 13, 928-942 (2014).

19. Yao, Y. et al. MiR-330-mediated regulation of SH3GL2 expression enhances malignant behaviors of glioblastoma stem cells by activating ERK and PI3K AKT signaling pathways. PLOS ONE 9, e95060 (2014).

20. Yoo, H. I., Kim, B. K. \& Yoon, S. K. MicroRNA-330-5p negatively regulates ITGA5 expression in human colorectal cancer. Oncol. Rep. 36, 3023-3029 (2016).

21. Trehoux, S. et al. Micro-RNAs miR-29a and miR-330-5p function as tumor suppressors by targeting the MUC1 mucin in pancreatic cancer cells. Biochim. Biophys. Acta 1853, 2392-2403 (2015).

22. Feng, L. et al. MiR-330-5p suppresses glioblastoma cell proliferation and invasiveness through targeting ITGA5. Biosci. Rep. 37, R20170019 (2017).

23. Lim, C. et al. Above $5 \mathrm{~cm}$, size does not matter anymore in patients with hepatocellular carcinoma. World J. Surg. 38, 2910-2918 (2014).

24. Allemann, P., Demartines, N., Bouzourene, H., Tempia, A. \& Halkic, N. Long-term outcome after liver resection for hepatocellular carcinoma larger than $10 \mathrm{~cm}$. World J. Surg. 37, 452-458 (2013).

25. Young, A. L. et al. Large hepatocellular carcinoma: time to stop preoperative biopsy. J. Am. Coll. Surg. 205, 453-462 (2007).

26. Zhang, H. et al. Tumor size does not independently affect long-term survival after curative resection of solitary hepatocellular carcinoma without macroscopic vascular invasion. World J. Surg. 38, 947-957 (2014).

27. Clarke, M. et al. Transcriptional regulation of SPROUTY2 by MYB influences myeloid cell proliferation and stem cell properties by enhancing responsiveness to IL-3. Leukemia 31, 957-966 (2017).

28. Chiu, Y. et al. Sprouty-2 regulates HIV-specific T cell polyfunctionality. J. Clin Invest. 124, 198-208 (2014).

29. Tsavachidou, D. et al. SPRY2 is an inhibitor of the ras/extracellular signalregulated kinase pathway in melanocytes and melanoma cells with wild-type BRAF but not with the V599E mutant. Cancer Res. 64, 5556-5559 (2004).

30. Cabrita, M. A. \& Christofori, G. Sprouty proteins, masterminds of receptor tyrosine kinase signaling. Angiogenesis 11, 53-62 (2008).

31. Matsumura, K. et al. Sprouty 2 controls proliferation of palate mesenchyma cells via fibroblast growth factor signaling. Biochem. Biophys. Res. Commun 404, 1076-1082 (2011).

32. $\mathrm{Xu}, \mathrm{Y}$. et al. Sprouty2 correlates with favorable prognosis of gastric adenocarcinoma via suppressing FGFR2-induced ERK phosphorylation and cancer progression. Oncotarget 8, 4888-4900 (2017).

33. Lito, P. et al. Relief of profound feedback inhibition of mitogenic signaling by RAF inhibitors attenuates their activity in BRAFV600E melanomas. Cancer Cell. 22, 668-682 (2012)

34. Sanchez, A. et al. Epigenetic inactivation of the ERK inhibitor Spryz in B-cell diffuse lymphomas. Oncogene 27, 4969-4972 (2008).

35. Fong, C. W. et al. Sprouty 2, an inhibitor of mitogen-activated protein kinase signaling, is down-regulated in hepatocellular carcinoma. Cancer Res. 66, 2048-2058 (2006).

36. Wang, C. et al. Inactivation of Spry2 accelerates AKT-driven hepatocarcinogenesis via activation of MAPK and PKM2 pathways. J. Hepatol. 57, 577-583 (2012).

37. Desideri, E., Cavallo, A. L. \& Baccarini, M. Alike but different: RAF paralogs and their signaling outputs. CELL 161, 967-970 (2015).

38. Shukla, A. et al. Sprouty 2: a novel attenuator of B-cell receptor and MAPK-Erk signaling in CLL. Blood 127, 2310-2321 (2016). 
39. Shojaee, S. et al. Erk negative feedback control enables Pre-B cell transformation and represents a therapeutic target in acute lymphoblastic leukemia. Cancer Cell. 28, 114-128 (2015).

40. $\mathrm{Yu}, \mathrm{Z}$. et al. The RAF-MEK-ERK pathway: targeting ERK to overcome obstacles to effective cancer therapy. Future Med. Chem. 7, 269-289 (2015).
41. Altman, D. G., McShane, L. M., Sauerbrei, W. \& Taube, S. E. Reporting recommendations for tumor marker prognostic studies (REMARK): explanation and elaboration. BMC Med. 10, 51 (2012).

42. Xia, L. et al. Overexpression of forkhead box C1 promotes tumor metastasis and indicates poor prognosis in hepatocellular carcinoma. Hepatology 57, 610-624 (2013). 